\title{
Calibrated Automated Thrombinography (CAT), a Tool to Identify Patients at Risk of Bleeding during Anticoagulant Therapy: A Systematic Review
}

\author{
Suzanne Zwaveling ${ }^{1,2}$ Saartje Bloemen ${ }^{2}$ Bas de Laat ${ }^{2}$ Hugo ten Cate ${ }^{1}$ Arina ten Cate-Hoek ${ }^{1}$
}

${ }^{1}$ Laboratory for Clinical Thrombosis and Hemostasis, Internal Medicine and Biochemistry, Maastricht University, Maastricht, The Netherlands

2 Synapse Research Institute, Maastricht, The Netherlands

TH Open 2018;2:e291-e302.
Address for correspondence Suzanne Zwaveling, MD, Department of Internal Medicine and Biochemistry, Maastricht University, PO Box 616, UNS 50, 6200 MD, Maastricht, The Netherlands (e-mail: S.zwaveling@maastrichtuniversity.nl).

\begin{abstract}
Keywords

- thrombin generation

- vitamin $\mathrm{K}$ antagonist

- bleeding

- oral anticoagulant

Background Bleeding is a feared adverse event during anticoagulant treatment. In patients on vitamin $\mathrm{K}$ antagonists, most bleedings occur with the international normalized ratio (INR) in the therapeutic range. Currently, identification of high-risk patients via laboratory methods is not reliable. In this systematic review, we assessed the ability of calibrated automated thrombin generation (CAT-TG) to predict bleeding in patients on anticoagulant treatment.

Methods A systematic search was executed in three databases: Medline, Embase, and Cochrane.

Results Seven studies were included; two were of good methodological quality. One study showed that patients on warfarin with INRs in range (2-3) admitted for hemorrhage $(n=28)$, had lower CAT-TG levels (endogenous thrombin potential [ETP]: $333 \pm 89 \mathrm{nM} / \mathrm{min}$ ) than patients on warfarin admitted for other reasons (ETP: $436 \pm 207 \mathrm{nM} / \mathrm{min} ; p<0.001$ ). Another study found no difference in ETP or peak levels between bleeding and nonbleeding patients in PPP or PRP. When measured in whole blood, both levels were significantly lower in patients with bleeding compared with nonbleeding patients (median [interquartile range, IQR] ETP: 182.5 [157.2-2,847 $\mathrm{nM} / \mathrm{min}$ ] vs. median [IQR] ETP: 256.2 [194.9-344.2 nM/min]; $p<0.001$ ) and median [IQR] peak: 23.9 [19.6-41.8 nM] vs. median [IQR] peak: 39.1 [24.9-53.2 nM]; $p<0.05)$. From the remaining studies, four suggested that CAT-TG is more sensitive in detecting hemostatic abnormalities than INR and one article found ETP and INR to be equally useful. However, insufficient data were provided to validate these conclusions. Conclusion Studies investigating the direct association between decreased CAT-TG values and hemorrhagic events are rare. Therefore, the clinical consequences of low CAT-TG values remain to be further investigated.
\end{abstract}

\section{Introduction}

Thromboembolic disease is a major cause of morbidity and mortality worldwide. The treatment of thromboembolism relies on anticoagulant drugs of which direct oral anticoagulants (DOACs) and vitamin K antagonists (VKAs) are the most widely prescribed. Although anticoagulant therapy has proven to be effective since many years, it is associated with serious adverse effects. Major bleeding is a feared, but prevalent, complication with an incidence of 1 to $3 \%$ annually. ${ }^{1}$ Patients on VKA are monitored by measuring the prothrombin time (PT), which is standardized to the international normalized
License terms

Stuttgart - New York

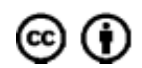

March 19, 2018 accepted after revision August 1, 2018
DOI https://doi.org/

10.1055/s-0038-1672183. ISSN 2512-9465. 
ratio (INR). The INR is used to adjust therapy toward therapeutic levels order to minimize the risk of bleeding. ${ }^{2}$ Nevertheless, clinical studies have shown that most bleeding complications occur within a therapeutic INR range. ${ }^{3-5}$ Rates of major bleedings between 3.09 and 3.4\% have been described in patients using warfarin with a time in therapeutic range (TTR) of 66\%; for cerebral hemorrhage, these numbers are approximately between 0.38 and $0.7 \%{ }^{6-8}$ For DOAC, the rates of major bleedings lie between 2.13 and $3.11 \%$, and rates of cerebral hemorrhage are between 0.1 and $0.5 \% .{ }^{6-8}$

Other than INR assessment, which at least helps reduce the risk of bleeding in patients using VKA, no other laboratory test has been helpful in limiting bleeding risk during anticoagulant therapy. The predictive value for bleeding of the PT and the activated partial thromboplastin time (aPTT) has been investigated in several studies, which all found poor correlations between these laboratory tests and hemorrhagic events. ${ }^{9-11}$ Furthermore, studies have shown that the PT and aPTT are unsuitable to measure the effect of DOAC. In contrast, global coagulation tests, such as calibrated automated thrombin generation (CAT-TG), are able to capture more aspects of the coagulation system, and could potentially be beneficial in assessing the total anticoagulant effects.

By this reasoning, some studies indeed suggested added value of CAT-TG testing in relation to bleeding outcome, ${ }^{12}$ or for monitoring of patients on anticoagulant therapy. ${ }^{13}$ CATTG is able to show the anticoagulant effect of many, possibly all, anticoagulants including DOACs. Multiple studies have previously found that patients who are treated with VKA have a diminished amount of thrombin that is formed over time (low endogenous thrombin potential [ETP]), a lower maximum of thrombin that is formed (low peak height), and a postponed start of coagulation (prolonged lag time) compared with healthy controls. ${ }^{14,15}$

We hypothesized that patients who are at risk of bleeding during anticoagulant therapy would have even lower CAT-TG values than patients using anticoagulants who are less at risk of bleeding. In this systematic review, we evaluate whether CAT-TG can detect the risk of bleeding in patients on oral anticoagulant treatment, and as such would be of potential clinical value.

\section{Methods}

\section{Data Sources and Searches}

We performed a systemic search for studies evaluating the use of CAT-TG to predict bleeding in patients on anticoagulant therapy in three databases (Medline, Embase, and Cochrane). The date of the search performed in Medline was May 18, 2017. On May 22, 2017, we systematically searched the Embase and Cochrane databases. No restrictions or filters with regard to language, publication date, and age were applied. The search strategy was refined using keywords of references found in a pilot search and after manual review of reference lists. The keywords included synonyms for "thrombin generation," "anticoagulant therapy," and "bleeding" as outcome (see -Supplementary Appendix for the complete search strategy). If an article was eligible for full-text reading but could not be retrieved, attempts were made to retrieve the article by searching other libraries or contacting authors. Authors were also contacted in case reports were available only as conference abstracts. The results of the database searches were supplemented by manual review of a reference list of articles that met the inclusion criteria. Duplicate articles were filtered manually.

\section{Study Selection}

Two reviewers independently screened abstracts between May 22, 2017, until July 2017 and selected articles for eligibility based on predefined inclusion and exclusion criteria. A third reviewer was consulted to agree on the final selection and to resolve any discrepancies between the first two reviewers. For a complete overview of the selection procedures, see - Fig. 1. We included studies that fulfilled the following criteria: (1) research was performed in patients using oral or parenteral anticoagulant treatment for more than 3 months; (2) CAT-TG was measured using a calibrated automated global TG test and the most common parameters: lag time (the time until the first thrombin is formed), peak (the maximum amount of thrombin that is formed), time to peak (time until the maximum is reached), and the ETP (the total amount of thrombin that can be formed over time); (3) there was a clear description of the method of CAT-TG, for example, noting the amount of tissue factor (TF), the use of corn trypsin inhibitor (CTI), thrombomodulin (TM) or activated protein $C$ (APC), the use of phospholipids, and the characteristics of the sample material (platelet-poor plasma [PPP], platelet-rich plasma [PRP], or whole blood); and (4) the outcome of CAT-TG was related to bleeding. Bleedings should have occurred spontaneously, that is, not by a procedure (e.g., postoperative bleeding or a punch biopsy). Additionally, bleeding episodes should have been well documented and described. When a study cohort was described by more than one article, we included only the original data.

Studies were excluded if they (1) consisted of non-original data (e.g., reviews, guidelines, comments); (2) were not written in English; (3) were performed in patients with a known bleeding disorder (e.g., hemophilia), severe liver cirrhosis or liver failure, cancer, or in pediatric patients; (4) were animal studies; (5) did not use the calibrated automated thrombinography (CAT) method as automated global thrombin generation test, but the Technoclone method, measured prothrombin fragment F1 and F2, or thrombin-antithrombin complexes; or (6) did not have "spontaneous clinical bleeding" as the outcome (e.g., postoperative bleeding or punch biopsy).

\section{Data Extraction and Quality Assessment}

The included studies were reviewed in duplicate and data were extracted using a standardized form. The extracted data included author, journal, year of publication, study design, clinical setting, number of patients, patient characteristics including the type of anticoagulant, the method of TG, other types of test used for comparison, bleeding events, follow-up, statistical analysis, and results. It was intended to construct $2 \times 2$ tables where possible, for the patients with a high/low risk of bleeding, using the extracted numbers of true and false 


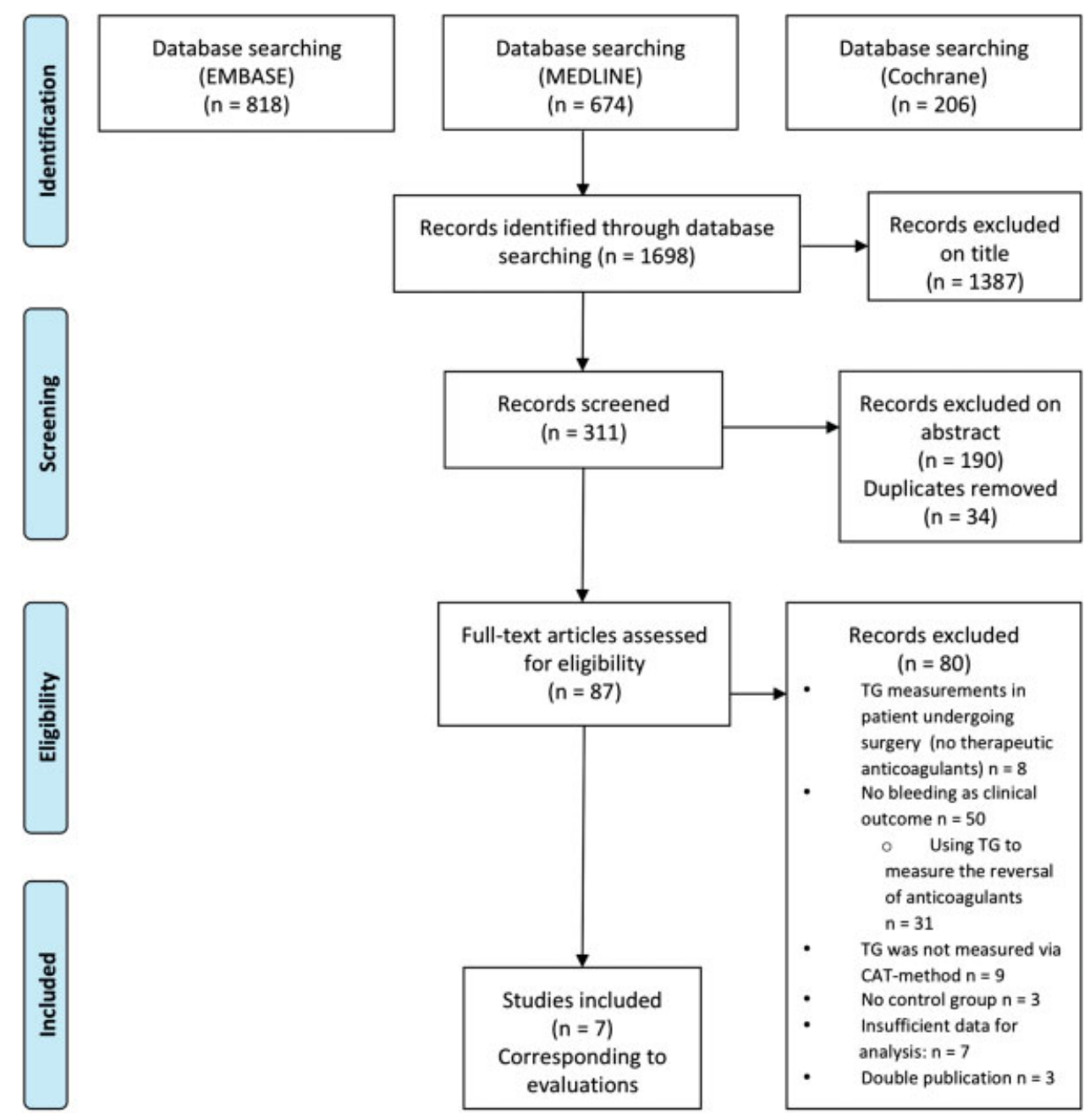

Fig. 1 Flow diagram of literature review.

positive as well as negative results according the TG test. Methodological quality of studies was assessed using the Newcastle-Ottawa quality assessment scale for cohort studies and case-control studies. To be able to classify the quality of the articles, we created a table in which we defined the amount of stars. Both are described in the -Supplementary Appendix. ${ }^{16}$

\section{Results}

\section{Search Results}

The database search yielded 1,698 articles in total: 674 articles were retrieved from Medline, 818 from Embase, and 206 from Cochrane ( - Fig. 1). A total of 1,387 articles were excluded based on title or abstract. Eighty-seven articles remained eligible and were evaluated based on full text. The majority of studies were not performed in patients on long-term anticoagulant treatment and/or did not assess the direct relation between CAT-TG and clinical bleeding in the absence of an intervention and were therefore excluded $(n=50)$. For example, 31 of these studies assessed TG as a tool to investigate the reversal of anticoagulation. The examined drugs in these studies were often DOACs and tests were mostly performed in healthy subjects. These studies used the normalization of coagulation tests (such as CAT-TG) as an indication that coagulation was restored, but did not investigate the relation with clinical bleeding. ${ }^{13,17-45}$ Eight studies investigated the role of TG in prediction of bleeding after cardiac surgery. ${ }^{46-53}$ These studies were excluded because all patients underwent surgical intervention; therefore, the outcome was not compatible with spontaneous hemorrhage $(n=8)$. We did not find any studies investigating DOACs and the direct relation between TG and clinical bleeding. Finally, seven articles were included. All articles studied the value of CAT-TG to evaluate a bleeding risk in patients using VKAs and two of these are currently unpublished. We approached the authors, but unfortunately no additional information could be retrieved.

\section{General Aspects and Results of the Included Studies}

The studies selected were mostly prospective studies. There are some similarities between the studies concerning the choice of anticoagulants, the target range for the INR, and the investigated method of TG. There was heterogeneity in patient selection, duration of follow-up, and also in the registration of outcome parameters. A general overview of the included studies is given in $\boldsymbol{- T a b l e} \mathbf{1}$. More specific data according CAT- 


\begin{tabular}{|c|c|c|c|c|c|c|}
\hline 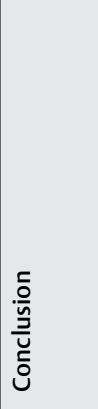 & 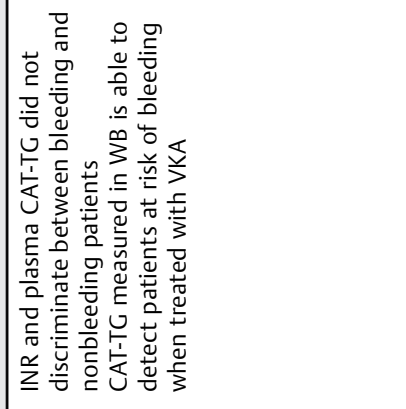 & 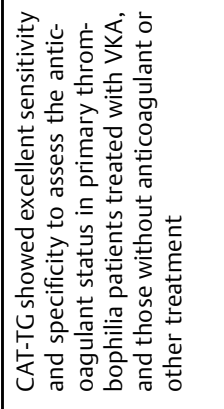 & 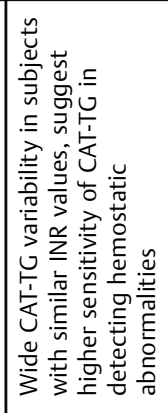 & 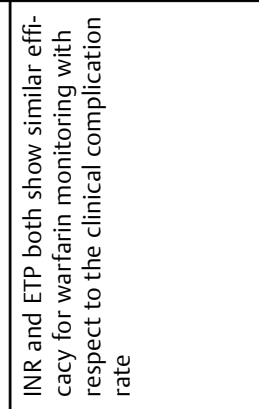 & 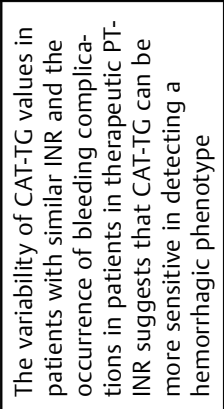 & 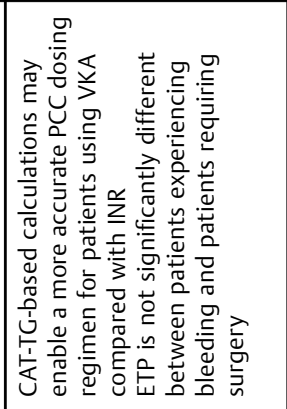 \\
\hline$\frac{n}{\vec{z}}$ & 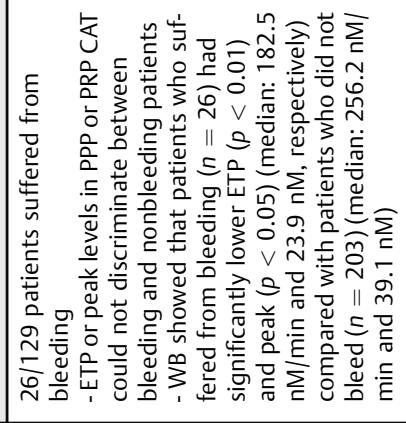 & 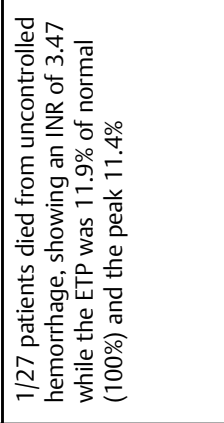 & 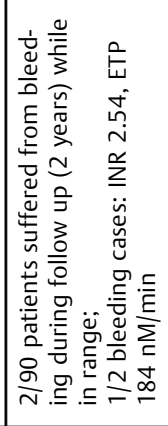 & 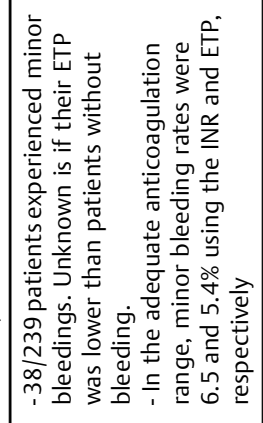 & 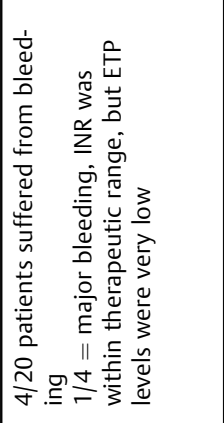 & 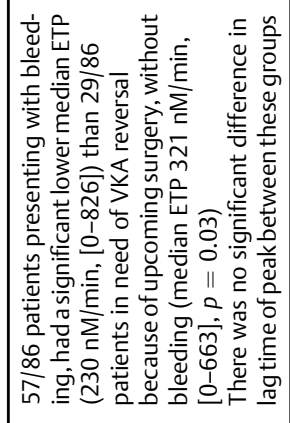 \\
\hline & 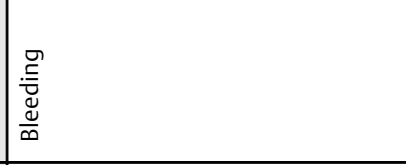 & 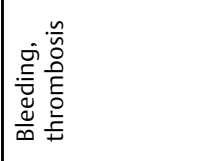 & 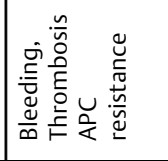 & 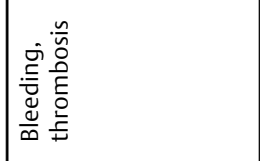 & 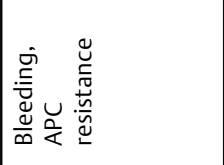 & 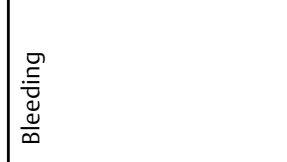 \\
\hline 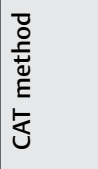 & 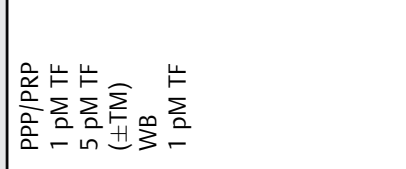 & 崖 & 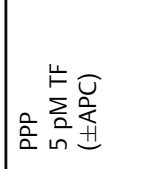 & 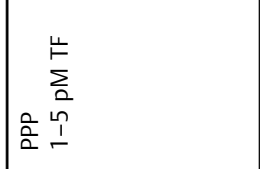 & 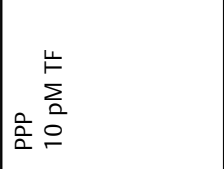 & 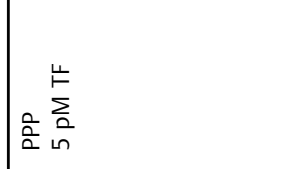 \\
\hline 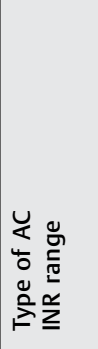 & 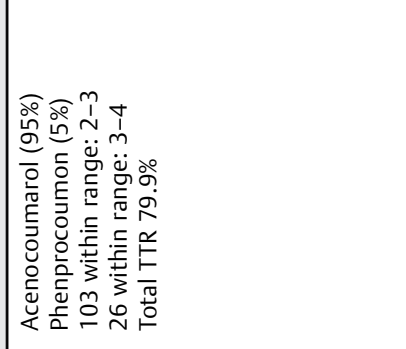 & 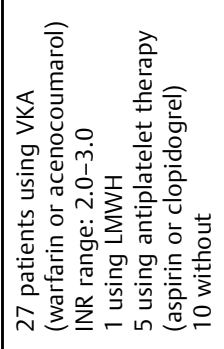 & 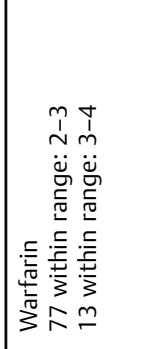 & 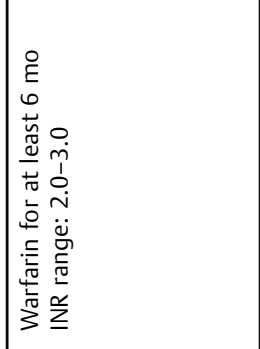 & 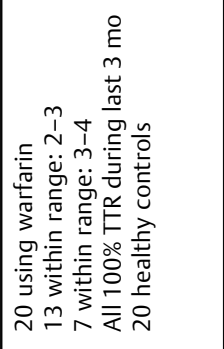 & 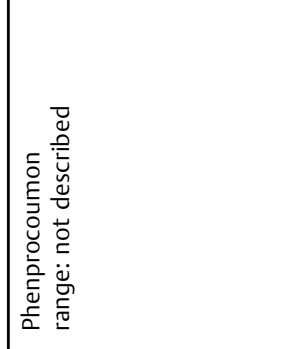 \\
\hline 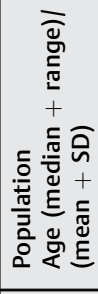 & 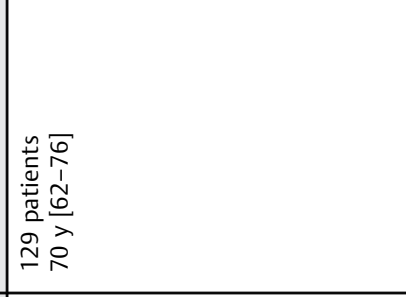 & 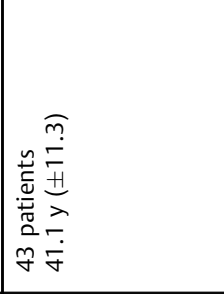 & 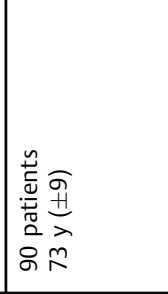 & 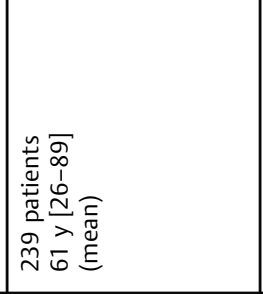 & 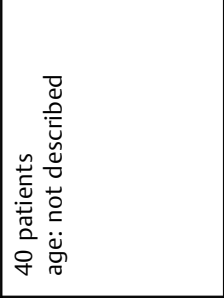 & 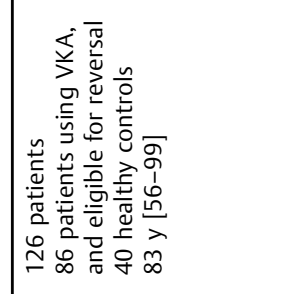 \\
\hline $\begin{array}{l}. \overline{5} \\
\frac{\bar{y}}{\square}\end{array}$ & 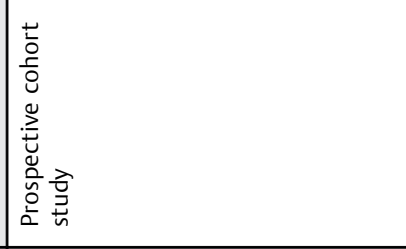 & 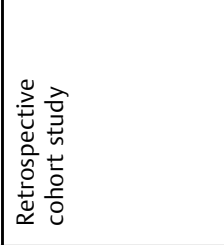 & 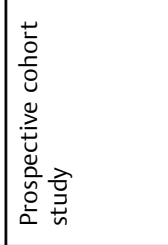 & 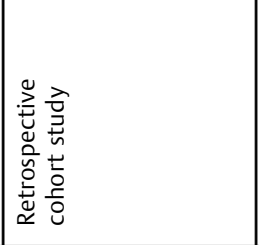 & 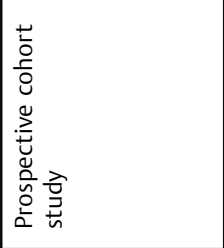 & 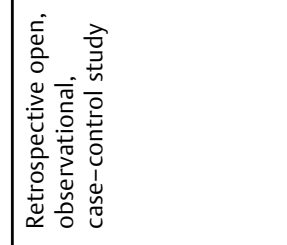 \\
\hline 离 & 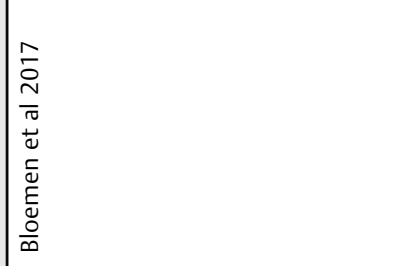 & 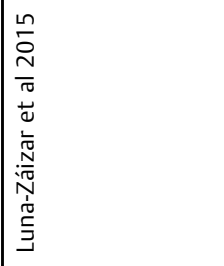 & 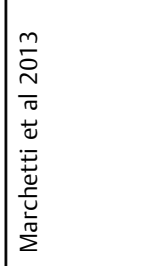 & 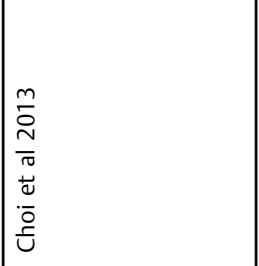 & 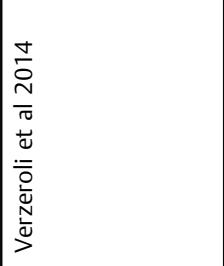 & 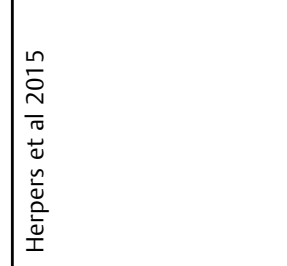 \\
\hline
\end{tabular}




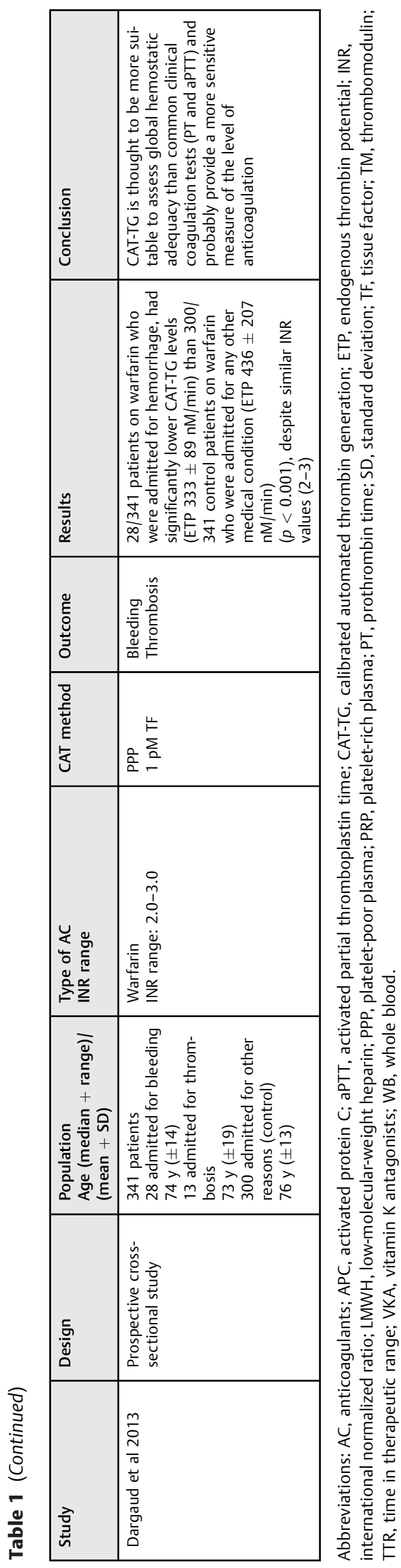

TG values are displayed in $\boldsymbol{-}$ Table 2 . The quality of the included studies was rated according to the Newcastle-Ottawa Quality assessment scale ( $\mathbf{- T a b l e s} \mathbf{3}$ and $\mathbf{4}$ ).

\section{Cohort Studies}

Bloemen et al studied whether CAT-TG either in plasma or in whole blood could be used to predict bleeding episodes in patients using VKA. ${ }^{54}$ The authors included 129 patients who used VKA for at least 3 months with an average age of 68 years. Of this population, $21.7 \%$ were female and $95.4 \%$ of the patients were treated with acenocoumarol. The average TTR was $79.9 \%$. Of the included patients, 103 were classified in the lower INR range (2.0-3.0) and 26 patients in the higher range (2.5-4.0). Patient characteristics were well defined and there was a follow-up of 15.5 months. As main outcome, clinically relevant bleeding episodes were scored during follow-up according to the criteria defined by the Dutch Federation of Anticoagulation Clinics (FNT). Clinically relevant bleeding episodes occurred in 26 (20.2\%) patients. A total of 44 bleeding episodes were registered, which is due to the fact that some patients had multiple episodes. CAT-TG measured in PPP with 5 pM TF showed lower ETP levels in patients who had experienced bleeding compared with patients without bleeding; however, this difference was not significant. Also other CAT-TG parameters like peak height could not discriminate between bleeding and nonbleeding patients $(p=0.18)$ nor could INR $(p=0.87)$, hematocrit $(p=0.23)$, hemoglobin $(p=0.11)$, or fibrinogen level $(p=0.54)$. In contrast, an increased HAS-BLED score was significantly associated with bleeding $(p<0.05)$. Interestingly, CAT-TG stimulated with $1 \mathrm{pM} \mathrm{TF}$ in whole blood yielded significantly lower ETP $(p<0.01)$ and peak height levels $(p<0.05)$ in patients that suffered from bleeding, compared with patients who did not bleed.

Luna-Záizar et al explored the usefulness of CAT-TG to assess the anticoagulation status compared with the INR in patients with primary thrombophilia. ${ }^{55}$ Fifty patients, who were diagnosed with inherited thrombophilia and had experienced at least one thrombotic event, were included. Whether the selection was consecutive is not described, neither are the criteria for exclusion. Definitions of the outcome "bleeding" (major or minor) were not further explained. Complete results were obtained from 43 patients (13 males [30\%]/30 females [70\%], mean age: $41.1 \pm 11.3$ years). Twenty-seven of these patients were treated with VKA and 1 patient was treated with low-molecular-weight heparin (LMWH). Fifteen patients were either without anticoagulation medication or used antiplatelet therapy only. CAT-TG was initiated with 5 pM TF in PPP. During the study, only one bleeding event occurred. This patient on VKA treatment suffered from fatal hemorrhage. While the INR was 3.47, ETP and peak levels were lower than CAT-TG values in patients using VKA (2.0-3.0) who did not bleed. Furthermore, the study showed significant lower ETP and peak values for patients who used VKA or LMWH compared with patients who were untreated $(p<0.0001)$. Lag time was also discriminative between these groups but displayed great interindividual variability in the group of patients under optimal anticoagulation with VKA (INR: 2.0-3.0). An inverse nonlinear 
Table 2 CAT-TG values compared in bleeding and nonbleeding patients using VKA

\begin{tabular}{|c|c|c|c|c|c|c|c|c|c|}
\hline \multirow{2}{*}{ Author } & \multirow[b]{2}{*}{ CAT-TG } & \multirow[b]{2}{*}{$\mathrm{N}$} & \multicolumn{4}{|l|}{ Bleeding } & \multicolumn{3}{|c|}{ Nonbleeding } \\
\hline & & & & Median/Mean & IQR/SD & $\mathrm{N}$ & $\begin{array}{l}\text { Median/ } \\
\text { Mean }\end{array}$ & IQR/SD & $p$-value \\
\hline \multirow[t]{8}{*}{ Bloemen et al } & WB & 26 & ETP (nM/min) & 182.5 & $157-285$ & 101 & 256.2 & 195-344 & $<0.01^{\mathrm{a}}$ \\
\hline & & & Peak (nM) & 23.9 & $20-42$ & & 39.1 & $25-53$ & $<0.05^{\mathrm{b}}$ \\
\hline & & & TtPeak (min) & 12.2 & $10-18$ & & 12.2 & $10-15$ & 0.427 \\
\hline & & & Lag time (min) & 7.1 & $5-8$ & & 6.5 & $5-8$ & 0.545 \\
\hline & PPP & 26 & ETP (nM/min) & 367 & $298-501$ & 101 & 438 & $344-541$ & 0.09 \\
\hline & & & Peak (nM) & 76 & 59-99 & & 86.6 & $63-114$ & 0.181 \\
\hline & & & TtPeak (min) & 7.8 & $6-10$ & & 8.3 & $7-10$ & 0.897 \\
\hline & & & Lag time (min) & 5.1 & $4-7$ & & 5.5 & $4-7$ & 0.805 \\
\hline \multirow[t]{3}{*}{ Luna-Záizar et al } & PPP & 1 & $\begin{array}{l}\text { ETP } \\
\% \text { of normal } \\
(\text { normal }=100 \%)\end{array}$ & $11.9 \%$ & & 26 & $31.2 \%$ & \pm 12 & $\mathrm{n} / \mathrm{a}$ \\
\hline & & & $\begin{array}{l}\text { Peak } \\
\% \text { of normal) } \\
\text { (normal = 100\%) }\end{array}$ & $11.4 \%$ & & & $32 \%$ & $\pm \mathbf{1 0}$ & $\mathrm{n} / \mathrm{a}$ \\
\hline & & & Lag time (min) & $\mathrm{n} / \mathrm{a}$ & & & 6 & \pm 2 & $\mathrm{n} / \mathrm{a}$ \\
\hline \multirow[t]{4}{*}{ Marchetti et al } & PPP & 2 & $\mathrm{ETP}(\mathrm{nM} / \mathrm{min})$ & $184^{c}$ & & 90 & 435 & \pm 190 & $\mathrm{n} / \mathrm{a}$ \\
\hline & & & Peak (nM) & 76 & & & 109 & \pm 51 & $\mathrm{n} / \mathrm{a}$ \\
\hline & & & TtPeak (min) & $\mathrm{n} / \mathrm{a}$ & & & 6 & \pm 2 & $\mathrm{n} / \mathrm{a}$ \\
\hline & & & Lag time (min) & $\mathrm{n} / \mathrm{a}$ & & & 4 & \pm 2 & $\mathrm{n} / \mathrm{a}$ \\
\hline Choi et al & PPP & 38 & & $\mathrm{n} / \mathrm{a}$ & & 239 & $\mathrm{n} / \mathrm{a}$ & & $n / a$ \\
\hline Verzeroli et al & PPP & 4 & & $\mathrm{n} / \mathrm{a}$ & & 20 & $\mathrm{n} / \mathrm{a}$ & & $\mathrm{n} / \mathrm{a}$ \\
\hline \multirow[t]{3}{*}{ Herpers et al } & PPP & 57 & ETP (nM/min) & 230 & $(0-826)$ & 29 & 321 & $(0-663)$ & $0.03^{\mathrm{b}}$ \\
\hline & & & Peak (nM) & 27 & $(0-208)$ & & 42 & $(0-145)$ & $>0.05$ \\
\hline & & & Lag time (min) & 12 & $(3$ to $>60)$ & & 8 & $(4$ to $>60)$ & $>0.05$ \\
\hline Dargaud et al & PPP & 28 & ETP (nM/min) & 333 & \pm 89 & 300 & 436 & \pm 207 & $<0.001^{\mathrm{a}}$ \\
\hline
\end{tabular}

Abbreviations: CAT-TG, calibrated automated thrombin generation; ETP, endogenous thrombin potential; IQR, interquartile range; $\mathrm{n} / \mathrm{a}$, not applicable; N, number; PPP, platelet-poor plasma; SD, standard deviation; TtPeak, time to peak; WB, whole blood; VKA, vitamin K antagonist. Note: Mean values with SD are in bold; the other values are medians with interquartile ranges.

${ }^{a}$ Significant difference, $p<0.01$.

'bignificant difference, $p<0.05$.

'Only CAT-TG values from one patient were reported, although two patients suffered from bleeding.

relation was found between ETP values $\left(R^{2}=0.649\right)$, peak $\left(R^{2}=0.633\right)$, and the velocity index $\left(R^{2}=0.532\right)$ versus INR values in patients treated with VKA. As expected, a positive linear correlation between lag time and INR was found $\left(R^{2}=0.338\right)$.

Marchetti et al performed a prospective cohort study to investigate the characteristics of CAT-TG in patients with atrial fibrillation $(\mathrm{AF})$ on permanent oral anticoagulation therapy with warfarin. ${ }^{56}$ They included 90 patients (56 males/34 females; aged $73 \pm 9$ years) of whom 77 patients had a target INR within 2.0 to 3.0 range, and 13 patients within 3.0 to 4.0 range. Other patient characteristics such as concomitant use of antiplatelet therapy were not presented. Outcomes of interest were bleeding and thrombosis, but predefined criteria for bleeding (minor or major) or thrombosis were not described in detail. Patients were followed up for an average time of 2 years. During follow-up, two bleed- ings occurred: one patient suffered a gastric bleeding and another patient experienced cerebral hemorrhage, both of them being within the INR target range. One of these patients with an INR of 2.54 did have a low ETP value. CAT-TG was performed using $5 \mathrm{pM}$ TF. Besides the ETP, other parameters were also evaluated: peak height, lag time, and time to peak. A significantly decreased ETP $(435 \pm 190$ vs. $1,229 \pm 114$ $\mathrm{nM} / \mathrm{min})$ and peak $(109 \pm 51$ vs. $256 \pm 44 \mathrm{nM})$ were observed in patients with stable INRs in their appropriate range, compared with healthy controls $(p<0.01)$. In addition, lag time and time to peak were significantly prolonged in the patient group ( $4.1 \pm 1.5$ vs. $2.0 \pm 0.3$ minutes and $6.2 \pm 1.7$ vs. $4.6 \pm 0.6$ minutes, respectively).

A moderate correlation between INR and all CAT-TG parameters $\left(R^{2}=0.6\right)$ was found, but patients with similar INRs showed a large variability in TG levels, particularly the patients within target INR range of 2.0 to 3.0. 
Thrombin Generation to Detect a Risk of Bleeding Zwaveling et al. e297
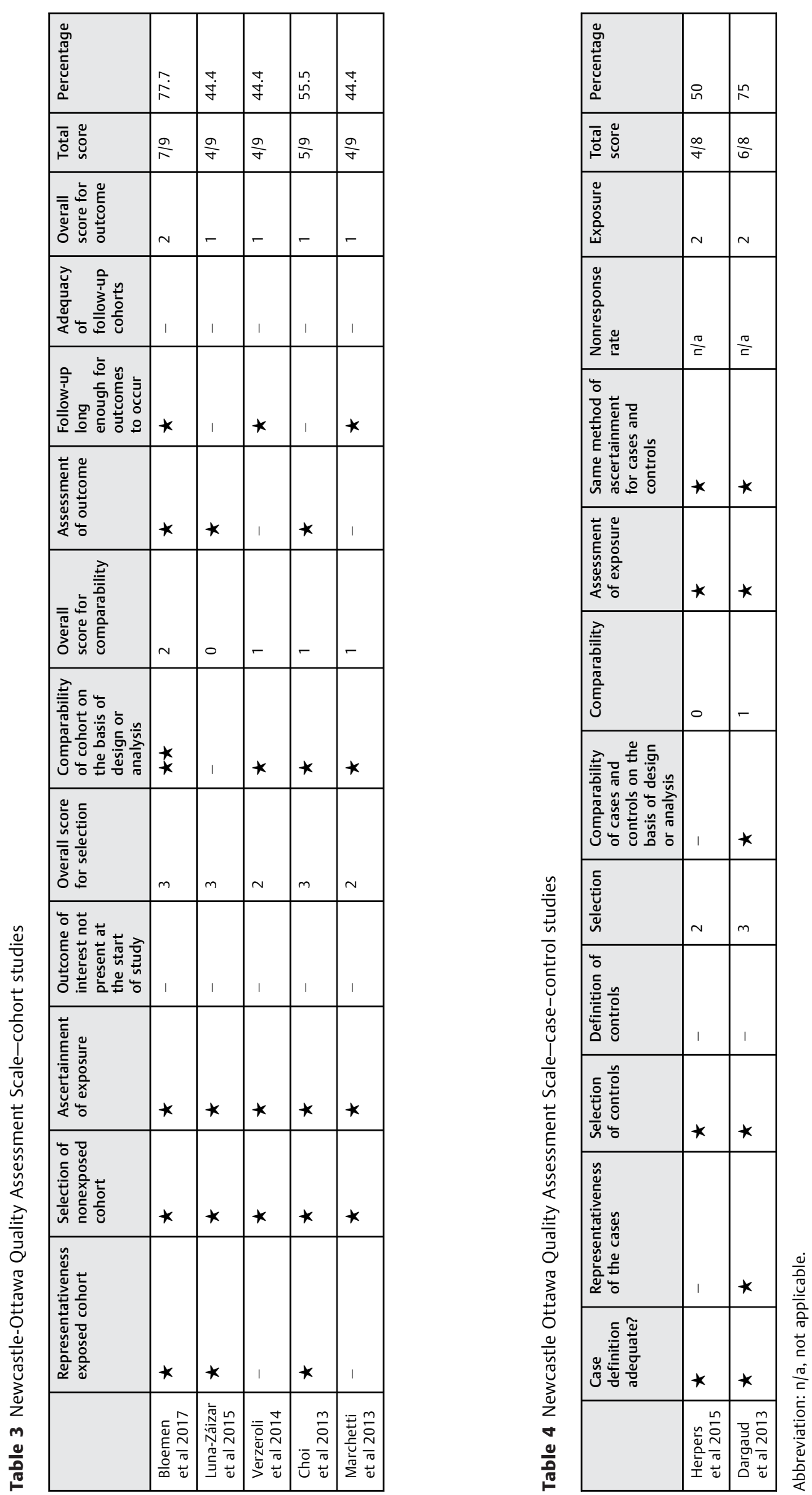
Choi et al compared the monitoring performance of ETP with INR. ${ }^{57}$ In this retrospective cohort study, 239 patients (129 males/110 females; mean age: 61 years, range: 2689 years) were included using warfarin for at least 6 weeks for several indications (prosthetic heart valves, $\mathrm{AF}$, ischemic heart failure, or DVT). The INR ranged from 1.0 to 4.26 . Exclusion criteria were younger than 19 years, the use of other anticoagulant treatment than warfarin, or the use of antiplatelet agents. Outcome was bleeding and thrombosis, obtained from medical records. TG was performed using the CAT method. The amount of TF and PL was not clearly mentioned, but a reference was made to the article of Hemker et al in which $5 \mathrm{pM} \mathrm{TF}$ and $4 \mu \mathrm{M}$ PL were used. ${ }^{58} \mathrm{~A}$ significant inverse correlation between ETP and INR was established $(r=-0.769$, $p<0.001)$. With a regression equation, the authors calculated a therapeutic range for the ETP (290.1-494.6 nM/min), which would correspond to the INR range from 2.0 to 3.0. Subsequently, the patients were divided into three groups (under-, adequately, and over-anticoagulated) according to their anticoagulation status judged by the INR or ETP. Whether the ETP is measured per individual or calculated based on the formula mentioned earlier remains uncertain. To assess the monitoring performance of the INR and ETP, they compared their anticoagulation status to clinical complications rates. When the division to the adequate anticoagulation group was based on INR or ETP, bleeding rates were 6.3 and $5.4 \%$, respectively. Although the use of ETP as target parameter yielded a lower bleeding rate, the difference was not statistically significant. During the study period, only minor bleedings occurred in 38 patients. Individual ETP values were not reported in the article.

Verzeroli et al investigated the correlation between CAT-TG and INR in patients treated with warfarin and whether CAT-TG could be useful to identify subjects at a higher risk of bleeding. ${ }^{59}$ The study included 20 patients using warfarin ( 13 for AF, 7 for cardiac valve prosthesis) who had been in their appropriate target INR ranges for 100\% over the last 3 months; 20 healthy subjects were studied as controls. Patient characteristics were not described, nor were INR target ranges specified. Patients were followed up for 1 year. CAT-TG when activated with $10 \mathrm{pM} \mathrm{TF}$, showed a significantly lower TG potential in the patients using warfarin compared with healthy controls; a significant correlation between the INR levels and all TG parameters ( $p<0.01$ ) was mentioned, but the actual data on correlations were not given. During follow-up, bleeding complications were registered in four patients, of which one was major, while all patients were in their respective target INR ranges. All four patients had a very low TG potential, but exact levels of CAT-TG parameters are not described. The diagnostic criteria of "bleeding" were not further defined.

\section{Case-Control Studies}

Herpers et al compared the use of the INR with CAT-TG to guide VKA reversal. ${ }^{35}$ In an open, observational study, they studied 86 patients treated with phenprocoumon, who were in need of VKA reversal. The median age was 83 years (range: 56-99 years), 53\% were female and the indication for VKA was mostly cardiac arrhythmia (84\%). They compared 29 patients who needed prophylactic VKA reversal because of upcoming surgery with 57 patients who needed VKA reversal because of hemorrhage. Coagulation reactions during CAT-TG were initiated with 5 pM TF. A significantly lower ETP was described in bleeding patients compared with nonbleeding patients. Lag time and peak did not differ significantly $(p>0.05)$ between these groups. However, in the Discussion and Conclusion sections, the authors stated that no significant difference was found in any CAT parameter (including the ETP).

Dargaud et al evaluated the hypothesis that the INR might underestimate the level of anticoagulation in patients with a lower factor IX level in contrast to CAT-TG, suggesting CAT-TG to be a more accurate test. ${ }^{60}$ In this study, 341 patients on warfarin with a stable INR between 2.0 and 3.0 were included at admission to the emergency department. Twenty-eight of these patients (18 males/10 females, aged $74 \pm 14$ years) were admitted for hemorrhage. Thirteen patients were admitted for thrombosis and were not taken into account in this review. Three hundred patients were admitted for other medical reasons (151 males/149 females, aged $76 \pm 13$ years). Of the 28 patients who were admitted for hemorrhage, 22 had major bleeding ( 7 spontaneous intracranial hemorrhage, 3 traumarelated intracranial hemorrhage, 12 with gastrointestinal tract bleeding); 5 patients exhibited muscle hematomas, severe epistaxis, or gum bleeding; and 1 patient had urinary tract bleeding. All patients were reviewed for potential underlying disorders that might explain their bleeding episodes, but no significant abnormalities were detected. The criteria for major bleeding were not explained in further detail. All CAT-TG experiments were activated with $1 \mathrm{pM}$ TF. Patients on warfarin who were admitted for hemorrhage $(n=28)$ had significantly lower TG levels than patients on warfarin who were admitted for any other medical condition, while having similar INRs in target range. No significant correlation between the INR levels in the range of 2.0 to 3.0, and ETP values $(r=-0.05,95 \% \mathrm{CI}$ : $=-0.168$ to $0.059 ; p=0.361$ ) was found. Mean TG levels of all admitted patients on warfarin with INRs in the target range of 2 to 3 ( $n=341$ ) were significantly lower than TG levels of 100 healthy controls (ETP $428 \pm 200 \mathrm{nM} / \mathrm{min}$ vs. ETP $1,380 \pm 214 \mathrm{nM} / \mathrm{min}$ ).

\section{Thrombin Generation Methods in the Included Studies} All studies measured TG by using the CAT method, although this method was not always adequately described. Bloemen et al, ${ }^{54}$ Luna-Záizar et al, ${ }^{55}$ Choi et al, ${ }^{57}$ Herpers et al, ${ }^{35}$ and Dargaud et $\mathrm{al}^{60}$ all used the CAT method propounded by Hemker et al. ${ }^{58}$ The assays were performed on a Fluorscan Ascent fluorometer (Thermo Labsystems OY, Helsinki, Finland). The software program, Thrombinoscope (Thrombinoscope BV), enabled the calculation of thrombin activity. Bloemen et al used $1 \mathrm{pM}$ as well as $5 \mathrm{pM}$ TF to initiate coagulation in PPP. Dargaud et al used 1 pM TF. ${ }^{60}$ Both LunaZáizar et $\mathrm{al}^{55}$ and Herpers et $\mathrm{al}^{35}$ used 5 pM TF, while Choi et $\mathrm{al}^{57}$ did not mention the used amount of TF. Bloemen et al measured CAT-TG also in whole blood, using $1 \mathrm{pM} \mathrm{TF}$, according the specifications of Ninivaggi et al. ${ }^{61}$ Marchetti et al and Verzeroli et al did not describe the CAT method in detail. They did report the amount of TF used, which was 5 and $10 \mathrm{pM}$, respectively. 
All studies used the same concentration of phospholipids $(4 \mu \mathrm{M})$. Two studies (Bloemen et $\mathrm{al}^{54}$ and Dargaud et $\mathrm{al}^{60}$ ) reported the specifications of the type of phospholipids used; both used phospholipids obtained from Avanti Polar Lipids (Alabaster, AL, United States).

\section{Discussion}

Hemorrhagic complications remain the most important concern during the management of anticoagulation therapy. Insight into the (individual) bleeding risk of patients would likely affect clinical decision making. However, sensitive instruments to identify patients at increased risk of bleeding, who would benefit from more careful management, are lacking. CAT-TG measures the total amount of thrombin formed over time, and offers a more global assessment of coagulation because it is influenced by all clotting factors involved in the cascade. ${ }^{62}$ Several studies have investigated the role of TG as a new tool to assess coagulation. ${ }^{13,15,63,64}$ This review was performed to assess the currently available evidence for the predictive ability of CAT-TG in relation to bleeding, not related to surgery, in patients on anticoagulant treatment.

We retrieved 1,698 articles when searching the key words "thrombin generation," "anticoagulant therapy," and "bleeding." Interestingly, most studies correlated CAT-TG to the INR, based on the known association between INR and risk of bleeding, to indirectly link bleeding risk to CAT-TG values. In our search, we also identified 31 articles exploring the value of TG for the assessment of the reversal of different anticoagulants, including the DOAC drugs. TG levels were used as reference point to determine whether coagulation was restored after adding three- or four-factor prothrombin complex concentrate (PCC), fresh frozen plasma (FFP), or factor VIII inhibitor bypass activity (FEIBA). ${ }^{13,17-42}$

Remarkably few studies investigated the direct relation between CAT-TG values and clinical bleeding in stable anticoagulated patients, and could therefore be included in this systematic review. Of these seven studies, only two studies were of good methodological quality. The quality of the other studies was considered as moderate. The data available from the studies were unfortunately insufficient to perform a meta-analysis. There are multiple techniques to measure TG. In this review, we chose to solely evaluate TG measured by the CAT method. The difference between methods, as well in protocol as in reagents, makes the TG data between techniques hard to compare. Since TG measured with CAT was previously found to be correlated to a hypocoagulable, as well as a hypercoagulable state in patients, we decided to investigate TG measures with CAT only, although this would limit our scope. ${ }^{51,65,66}$ Moreover, to fairly compare outcomes between different studies, a standardized protocol of the CAT method is important. Although the protocols of the included study were largely identical, two studies used different amounts of TF in CAT, which made comparison between outcomes of studies difficult. The use of lower amounts of TF (1 pM) will enhance the sensitivity of the assay, but could reduce the reproducibility. Choosing a high concentration of
TF (e.g., $10 \mathrm{pM}$ ), the contribution of the intrinsic pathway to coagulation will become negligible. This review stressed the need for an unambiguous study protocol for CAT-TG.

Some of the included studies were limited because of a small sample size. Additionally, in all included cohort studies, the number of patients who suffered from bleeding during the follow-up was lower compared with the patients who did not bleed. Consequently, the number of bleeding events may have been too low to reach solid conclusions about the predictive value of CAT-TG for bleeding.

The study by Dargaud et al found significantly lower mean ETP levels in patients experiencing warfarin-related hemorrhage compared with patients with similar INR values in the desired range, who did not experience bleeding. This study had sufficient power and enough clinical bleedings (8.2\%) to point out significant differences in ETP levels between bleeding and nonbleeding patients. However, the design of this study may not be suitable to answer the question whether low CAT-TG values are predictive for bleeding. In this crosssectional study, the patients suffered from warfarin-related hemorrhage at the time of inclusion and blood withdrawal for the study assessment. Therefore, the ETP levels most likely represent a more acute hemostatic condition, involving bleeding-associated consumption of coagulation proteins.

Herpers et al investigated whether patients in need of VKA reversal because of bleeding had lower ETP than patients in need of VKA reversal who did not bleed. ${ }^{35}$ This study was considered of moderate methodological quality, even more so because in the discussion and the conclusions of the article, contradictory interpretations of the findings were presented. In addition, the difference in design makes it difficult to compare the results of previous studies with the other included studies, which were cohort studies. In the cohort studies, the ETP levels are measured at inclusion while patients are in a stable, nonbleeding state, and bleedings are registered during follow-up. The latter design is more informative on the predicting ability of CAT-TG. Unfortunately, out of the five included prospective cohort studies, only two studies were of acceptable methodological quality. Both the studies of Marchetti et al and Vezeroli et al suggested that the differences in ETP values found in patients with similar INRs indicate that CAT-TG is more sensitive in detecting hemostatic abnormalities than INR. However, data were derived from conference abstracts only; therefore, information was incomplete. Luna-Záizar et al examined the use of CAT-TG in patients using VKA to assess the anticoagulation status compared with the INR, which corresponds to our research question..$^{55}$ The quality of the study was rated as moderate. The sample size was small and only one (major) bleeding event was described during follow-up. This patient had lower ETP values compared with other patients with similar INRs but did not bleed. The authors state that this clinical event suggests that the ETP can predict an increased hemorrhagic risk, making CAT-TG a better monitoring parameter than the INR. However, these conclusions are based on a single event rendering the outcome highly questionable. The research question of Choi et al was also in accordance with ours. ${ }^{57}$ The sample size of this study was sufficient and 
patients as well as controls were drawn out of the community. At the same time, the follow-up was poorly described. Moreover, the individual ETP values of patients could not be extracted from the data, making it impossible to compare ETP between bleeding and nonbleeding VKA patients. Patients were divided into groups based on their INR or ETP values. The calculation made to develop a therapeutic range for ETP, which should compare with the INR range 2.0 to 3.0, was partly based on the INR. Although the article shows an inverse correlation between INR and ETP, which is linear between the INR range 2.0 and 3.0, it cannot be assumed that this correlation will remain linear over a larger range. ${ }^{15,67}$ Therefore, ETP values comparable with INRs outside the range 2.0 to 3.0 cannot be justified. Overall, the results of this study were not suitable to answer our research question mainly because of the design despite the general moderate quality. The study by Bloemen et al was considered of fairly good methodological quality. The design was suitable to answer our research question and gave a detailed description of inclusion criteria, patient characteristics, and the specific execution of the CAT-TG test. Although lower average ETP levels were seen in the patients who experienced bleeding compared with patients who did not, no significant difference was observed in the PPP CAT-TG. It could be argued that this study was underpowered, and therefore not able to answer the hypothesis if CAT-TG can predict bleeding in patients. When a larger number of patients would have been investigated, a significant difference might be found, but a substantial overlap in ETP values between groups will remain, rendering CAT-TG in PPP probably not discriminative between bleeding and nonbleeding patients. One of the limitations of this study was a lack of correction for several confounding factors that are associated with a higher bleeding risk, for example, diabetes mellitus, a reduced kidney function, or a positive bleeding history. Additionally, the occurrence of bleeding complications during follow-up was higher than expected $(20.2 \%)$ and most bleedings were clinically relevant minor bleeds. Previous studies have shown that minor bleedings during anticoagulant therapy can be predictive for subsequent major bleedings, although the underlying causal mechanism for this is still to be elucidated. ${ }^{68,69}$ Some evidence indicates that minor bleedings are a marker for fixed and currently unknown risk factors for major bleeding events. In the study by Bloemen et al it was shown that CAT-TG measured in whole blood was able to distinguish patients at risk of bleeding. One explanation for these findings could be the concurrent effect of platelets and red cells in the whole blood thrombin generation assay. Since a previous study from our laboratory did not detect any significant influence of platelets on bleeding risk in anticoagulated patients, by demonstrating in PRP that platelet function tests and von Willebrand factor levels did not differ between bleeding and nonbleeding patients on $\mathrm{VKA}^{70}$ a contribution from red blood cells or leukocytes is likely.

Although the findings of Bloemen et al in whole blood are promising, whole blood TG is only recently developed, and the present study provides the first implementation in patients on VKA. More studies are needed to see if these results can be confirmed.

To genuinely evaluate the predictive ability of CAT-TG in relation to bleeding events, it is required to investigate the sensitivity, specificity, and positive and negative predictive value of the assay, to calculate an odds ratio or construct a receiver operation characteristic (ROC) curve. Unfortunately, this was not possible due to the absence of predefined cutoff values of CAT-TG parameters and because most articles compared mean or median CAT-TG values between patients who suffered from bleeding and patients who did not. Individual CAT-TG values of bleeding and nonbleeding patients were not reported.

Unfortunately, no articles were found investigating the value of CAT-TG to predict bleeding in patients using the DOACs. This could be explained by different reasons. First, measuring direct IIa inhibitors with TG shows a paradoxical increase of the peak and ETP and is therefore currently not reliable enough. ${ }^{71,72}$ Another explanation could be that measurements of direct Xa inhibitors, although possible with CAT-TG, have not yet been correlated to clinical bleeding complications in humans.

\section{Conclusion}

CAT-TG is frequently used to assess the effects of anticoagulation in different research settings. Clinical studies are mostly performed in patients treated with VKA, showing decreased TG values in patients compared with healthy controls. ${ }^{14,58,60,73}$

The studies of Bloemen et al and Dargaud et al, which were found to be of the highest methodological quality according this review, both found evidence supporting an association between low CAT-TG values and bleeding in patients using VKA. Other reviewed studies agreed with this reasoning, but did not provide enough data to validate this hypothesis. Unfortunately, studies investigating the direct association between decreased CAT-TG values and actual hemorrhagic events are scarce; therefore, the clinical consequences of low CAT-TG values remain to be investigated. To further evaluate whether low CAT-TG values can identify patients with a higher bleeding risk, new studies are needed.

While application in VKA treatment is an interesting avenue to pursue, it may be even more important to look for associations between CAT-TG activity and bleeding outcomes in patients using DOACs. The use of fixed doses of DOAC, based solely on patient characteristics, causes a wide variability in their anticoagulant responses, which makes testing with overall assays like CAT-TG potentially interesting.

\section{Conflicts of Interest}

H.t.C. is consultant to Stago, manufacturer of the CAT; he is also chair of the board of the Dutch Federation of Anticoagulation Clinics. All other authors declare to have no conflicts of interest. S.Z. and S.B. are linked to and B.d.L. is employed at Synapse Research Institute, which is part of Stago. 


\section{References}

1 Ansell J, Hirsh J, Hylek E, Jacobson A, Crowther M, Palareti G. Pharmacology and management of the vitamin $\mathrm{K}$ antagonists: American College of Chest Physicians Evidence-Based Clinical Practice Guidelines (8th Edition). Chest 2008;133(6, Suppl):160S-198S

2 Veeger NJ, Piersma-Wichers M, Tijssen JG, Hillege HL, van der Meer J. Individual time within target range in patients treated with vitamin $\mathrm{K}$ antagonists: main determinant of quality of anticoagulation and predictor of clinical outcome. A retrospective study of 2300 consecutive patients with venous thromboembolism. Br J Haematol 2005;128(04):513-519

3 Palareti G, Leali N, Coccheri S, et al; Italian Study on Complications of Oral Anticoagulant Therapy. Bleeding complications of oral anticoagulant treatment: an inception-cohort, prospective collaborative study (ISCOAT). Lancet 1996;348(9025):423-428

4 Jackson SL, Peterson GM, Vial JH, Daud R, Ang SY. Outcomes in the management of atrial fibrillation: clinical trial results can apply in practice. Intern Med J 2001;31(06):329-336

5 Abdelhafiz AH, Wheeldon NM. Results of an open-label, prospective study of anticoagulant therapy for atrial fibrillation in an outpatient anticoagulation clinic. Clin Ther 2004;26(09):1470-1478

6 Connolly SJ, Ezekowitz MD, Yusuf S, et al; RE-LY Steering Committee and Investigators. Dabigatran versus warfarin in patients with atrial fibrillation. N Engl J Med 2009;361(12):1139-1151

7 Patel MR, Mahaffey KW, Garg J, et al; ROCKET AF Investigators. Rivaroxaban versus warfarin in nonvalvular atrial fibrillation. $\mathrm{N}$ Engl J Med 2011;365(10):883-891

8 Granger CB, Alexander JH, McMurray JJ, et al; ARISTOTLE Committees and Investigators. Apixaban versus warfarin in patients with atrial fibrillation. N Engl J Med 2011;365(11):981-992

9 Chee YL, Crawford JC, Watson HG, Greaves M; British Committee for Standards in Haematology. Guidelines on the assessment of bleeding risk prior to surgery or invasive procedures. $\mathrm{Br} \mathrm{J}$ Haematol 2008;140(05):496-504

10 Suchman AL, Mushlin AI. How well does the activated partial thromboplastin time predict postoperative hemorrhage? JAMA 1986;256(06):750-753

11 Kitchens CS. To bleed or not to bleed? Is that the question for the PTT?. J Thromb Haemost 2005;3(12):2607-2611

12 Al Dieri R, Peyvandi F, Santagostino E, et al. The thrombogram in rare inherited coagulation disorders: its relation to clinical bleeding. Thromb Haemost 2002;88(04):576-582

13 Gatt A, van Veen JJ, Woolley AM, Kitchen S, Cooper P, Makris M. Thrombin generation assays are superior to traditional tests in assessing anticoagulation reversal in vitro. Thromb Haemost 2008;100(02):350-355

14 Altman R, Scazziota A, Herrera L, González C. Relationship between thrombin generation and international normalized ratio in patients receiving oral vitamin $\mathrm{K}$ antagonist therapy. J Thromb Haemost 2007;5(07):1552-1569

15 Gerotziafas GT, Dupont C, Spyropoulos AC, et al. Differential inhibition of thrombin generation by vitamin $\mathrm{K}$ antagonists alone and associated with low-molecular-weight heparin. Thromb Haemost 2009;102(01):42-48

16 Wells G, Shea B, O'Connel D, et al. The Newcastle-Ottawa scale (NOS) for assessing the quality of nonrandomised studies in metaanalyses. Available at: http://www.ohri.ca/programs/clinical_epidemiology/oxford.asp

17 Desmurs-Clavel H, Huchon C, Chatard B, Negrier C, Dargaud Y. Reversal of the inhibitory effect of fondaparinux on thrombin generation by rFVIIa, aPCC and PCC. Thromb Res 2009;123(05):796-798

18 Zahir H, Brown KS, Vandell AG, et al. Edoxaban effects on bleeding following punch biopsy and reversal by a 4-factor prothrombin complex concentrate. Circulation 2015;131(01):82-90

19 Herrmann R, Thom J, Wood A, Phillips M, Muhammad S, Baker R. Thrombin generation using the calibrated automated thrombinoscope to assess reversibility of dabigatran and rivaroxaban. Thromb Haemost 2014;111(05):989-995
20 Levi M, Moore KT, Castillejos CF, et al. Comparison of three-factor and four-factor prothrombin complex concentrates regarding reversal of the anticoagulant effects of rivaroxaban in healthy volunteers. J Thromb Haemost 2014;12(09):1428-1436

21 Perzborn E, Heitmeier S, Laux V, Buchmüller A. Reversal of rivaroxaban-induced anticoagulation with prothrombin complex concentrate, activated prothrombin complex concentrate and recombinant activated factor VII in vitro. Thromb Res 2014;133 (04):671-681

22 Dinkelaar J, Patiwael S, Harenberg J, Leyte A, Brinkman HJ. Global coagulation tests: their applicability for measuring direct factor Xa- and thrombin inhibition and reversal of anticoagulation by prothrombin complex concentrate. Clin Chem Lab Med 2014;52 (11):1615-1623

23 Dargaud Y, Desmurs-Clavel H, Marin S, Bordet JC, Poplavsky JL, Negrier C. Comparison of the capacities of two prothrombin complex concentrates to restore thrombin generation in plasma from orally anticoagulated patients: an in vitro study. J Thromb Haemost 2008;6(06):962-968

24 Marlu R, Hodaj E, Paris A, Albaladejo P, Cracowski JL, Pernod G. Effect of non-specific reversal agents on anticoagulant activity of dabigatran and rivaroxaban: a randomised crossover ex vivo study in healthy volunteers. Thromb Haemost 2012;108(02):217-224

25 Skolnick BE, Mathews DR, Khutoryansky NM, Pusateri AE, Carr ME. Exploratory study on the reversal of warfarin with rFVIIa in healthy subjects. Blood 2010;116(05):693-701

26 Eerenberg ES, Kamphuisen PW, Sijpkens MK, Meijers JC, Buller HR, Levi M. Reversal of rivaroxaban and dabigatran by prothrombin complex concentrate: a randomized, placebo-controlled, crossover study in healthy subjects. Circulation 2011;124(14): 1573-1579

27 KhooTL, Weatherburn C, Kershaw G, Reddel CJ, Curnow J, Dunkley S. The use of FEIBA $®$ in the correction of coagulation abnormalities induced by dabigatran. Int J Lab Hematol 2013;35(02):222-224

28 Perlstein I, Wang Z, Song Y, et al. Reversal of apixaban anticoagulation by 4 -factor prothrombin complex concentrates in healthy subjects. Blood 2014;124(21):345

29 Evans G, Luddington R, Baglin T. Beriplex P/N reverses severe warfarin-induced overanticoagulation immediately and completely in patients presenting with major bleeding. $\mathrm{Br} \mathrm{J}$ Haematol 2001;115(04):998-1001

30 Cheung KC, Rodgers S, McRae S. Local experience in reversal dabigatran using activated prothrombin complex concentrates (FEIBA). Haematologica 2012;97:185

31 Brinkman HJM, Dinkelaar J, Patiwael S, Harenberg J, Leyte A. How should we measure the reversal of direct factor XA or thrombin inhibitors by prothrombin complex concentrate? J Thromb Haemost 2014;12:16

32 Reijers J, Moerland M, Frieling J, et al. Pharmacodynamic effects of two recombinant FVIIa products in anticoagulated healthy volunteers. J Thromb Haemost 2013;11:1129

33 Godier A, Martin AC, Samama CM, Fischer AM, Le Bonniec B. Reversal of anticoagulant effects of apixaban with non-specific prohaemostatic agents: an in vitro study. J Thromb Haemost 2013;11:826

34 Brinkman HJA, Herpers R, Van Beem RT, et al. Ex vivo monitoring of Fenprocoumon reversal by prothrombin complex concentrate using thrombography. J Thromb Haemost 2013;11:825

35 Herpers R, van Rossum AP, van Beem RT, et al. INR vs. thrombin generation assays for guiding VKA reversal: a retrospective comparison. Clin Chem Lab Med 2015;53(08):1227-1236

36 Mullier F, Douxfils J, Chatelain C, Chatelain B, Dogne JM. Thrombin generation assay is superior to traditional coagulometric and chromogenic assays to monitor new oral anticoagulants. Blood 2012;120(21):1169

37 Galan AM, Arellano-Rodrigo E, Veronica Sanz V, et al. Reversal of the antithrombotic action of rivaroxaban and dabigatran: a clinical study in healthy volunteers. Blood 2012;120(21):2261 
38 Pillitteri D, Pilgrimm-Thorp AK, Krause M, Scholz T, Kirchmaier C. Antidotal effects of non-specific reversal agents on anticoagulantinduced inhibition of thrombin generation. J Thromb Haemost 2013;11:562-563

39 Halim AB, Li Y, Stein E, Mendell J. Low concentrations of rhFVIIa or FEIBA significantly and rapidly reverse the anticoagulant effects of supratherapeutic edoxaban. Blood 2011;118(21):1252

40 Mullier F, Douxfils J, Robert S, et al. Dabigatran: safety, usefulness and practical details of drug monitoring.J Thromb Haemost 2011; 9:857

41 Adamidou D, Riddell A, Aghighi S, Bates S, Chowdary P. PRP thrombin generation (TG) in patients on oral anticoagulation with warfarin: Influence of tissue factor (TF) concentration and corn trypsin inhibitor (CTI). J Thromb Haemost 2011;9:590

42 Voils SA, Martin EJ, Mohammed BM, Bayrlee A, Brophy DF. Laboratory assessment of warfarin reversal with global coagulation tests versus international normalized ratio in patients with intracranial bleeding. Blood Coagul Fibrinolysis 2015;26(04): 443-447

43 Schultz NH, Tran HTT, Bjørnsen S, Henriksson CE, Sandset PM, Holme PA. The reversal effect of prothrombin complex concentrate (PCC), activated PCC and recombinant activated factor VII against anticoagulation of Xa inhibitor. Thromb J 2017;15:6

44 Calmette L, Martin AC, Le Bonniec B, et al. Ticagrelor reversal: in vitro assessment of four haemostatic agents. J Clin Pathol 2017; 70(09):733-739

45 Cheung YW, Barco S, Hutten BA, Meijers JC, Middeldorp S, Coppens $M$. In vivo increase in thrombin generation by fourfactor prothrombin complex concentrate in apixaban-treated healthy volunteers. J Thromb Haemost 2015;13(10):1799-1805

46 Davidson SJ, Burman JF, Philips SM, et al. Correlation between thrombin potential and bleeding after cardiac surgery in adults. Blood Coagul Fibrinolysis 2003;14(02):175-179

47 Radulovic V, Hyllner M, Ternström L, et al. Sustained heparin effect contributes to reduced plasma thrombin generation capacity early after cardiac surgery. Thromb Res 2012;130(05): 769-774

48 Schols SE, van der Meijden PE, van Oerle R, Curvers J, Heemskerk JW, van Pampus EC. Increased thrombin generation and fibrinogen level after therapeutic plasma transfusion: relation to bleeding. Thromb Haemost 2008;99(01):64-70

49 Schols SE, Lancé MD, Feijge MA, et al. Impaired thrombin generation and fibrin clot formation in patients with dilutional coagulopathy during major surgery. Thromb Haemost 2010;103 (02):318-328

50 Coakley M, Hall JE, Evans C, et al. Assessment of thrombin generation measured before and after cardiopulmonary bypass surgery and its association with postoperative bleeding.J Thromb Haemost 2011;9(02):282-292

51 Bosch YP, Al Dieri R, ten Cate $\mathrm{H}$, et al. Measurement of thrombin generation intra-operatively and its association with bleeding tendency after cardiac surgery. Thromb Res 2014;133(03): 488-494

52 Percy CL, Jones M, Mehta D, et al. Thrombin generation and postoperative bleeding in patients undergoing surgery requiring cardiopulmonary bypass. J Thromb Haemost 2013;11:578

53 Feder S, Siegemund A, Correia C, et al. Clinical application of thrombin generation: a deeper reflection of plasmatic haemostasis of ventricular assist device recipients. Thorac Cardiovasc Surg 2015;63(Suppl 1):OP56

54 Bloemen S, Zwaveling S, Ten Cate H, Ten Cate-Hoek A, de Laat B. Prediction of bleeding risk in patients taking vitamin $\mathrm{K}$ antagonists using thrombin generation testing. PLoS One 2017;12(05): e0176967

55 Luna-Záizar H, González-Moncada AI, Padilla-López EL, et al. Thrombin generation and international normalized ratio in inherited thrombophilia patients receiving thromboprophylactic therapy. Thromb Res 2015;136(06):1291-1298
56 Marchetti M, Giaccherini C, Lerede T, Maggioni A, Cefis M, Falanga A. Characterization of thrombin generation (TG) by the calibrated automated thrombography (CAT) in atrial fibrillation (AF) patients on permanent oral anticoagulation therapy (OAT) with warfarin. J Thromb Haemost 2013;11(Suppl 3):59-60

57 Choi Q Kim JE, Hyun J, Han KS, Kim HK. Contributions of procoagulants and anticoagulants to the international normalized ratio and thrombin generation assay in patients treated with warfarin: potential role of protein $\mathrm{Z}$ as a powerful determinant of coagulation assays. Thromb Res 2013;132(01):e70-e75

58 Hemker HC, Giesen P, Al Dieri R, et al. Calibrated automated thrombin generation measurement in clotting plasma. Pathophysiol Haemost Thromb 2003;33(01):4-15

59 Verzeroli C, Giaccherini C, Marchetti M, Milesi V, Barcella L, Falanga A. Thrombin generation (TG) potential in patients on chronic oral anticoagulant therapy (OAT). Thromb Res 2014;2:S98-S99

60 Dargaud Y, Hoffman M, Lefrapper L, et al. Bleeding risk in warfarinized patients with a therapeutic international normalized ratio: the effect of low factor IX levels. J Thromb Haemost 2013;11(06):1043-1052

61 Ninivaggi M, Apitz-Castro R, Dargaud Y, de Laat B, Hemker HC Lindhout $\mathrm{T}$. Whole-blood thrombin generation monitored with a calibrated automated thrombogram-based assay. Clin Chem 2012;58(08):1252-1259

62 Hemker HC, Al Dieri R, De Smedt E, Béguin S. Thrombin generation, a function test of the haemostatic-thrombotic system. Thromb Haemost 2006;96(05):553-561

63 van Veen JJ, Gatt A, Makris M. Thrombin generation testing in routine clinical practice: are we there yet? $\mathrm{Br} J$ Haematol 2008; 142(06):889-903

64 Curvers J, Thomassen MC, Rimmer J, et al. Effects of hereditary and acquired risk factors of venous thrombosis on a thrombin generation-based APC resistance test. Thromb Haemost 2002;88(01):5-11

65 Besser M, Baglin C, Luddington R, van Hylckama Vlieg A, Baglin T. High rate of unprovoked recurrent venous thrombosis is associated with high thrombin-generating potential in a prospective cohort study. J Thromb Haemost 2008;6(10):1720-1725

66 Tripodi A, Legnani C, Chantarangkul V, Cosmi B, Palareti G, Mannucci PM. High thrombin generation measured in the presence of thrombomodulin is associated with an increased risk of recurrent venous thromboembolism. J Thromb Haemost 2008;6 (08):1327-1333

67 Gatt A, van Veen JJ, Bowyer A, et al. Wide variation in thrombin generation in patients with atrial fibrillation and therapeutic International Normalized Ratio is not due to inflammation. $\mathrm{Br}$ J Haematol 2008;142(06):946-952

68 Veeger NJ, Piersma-Wichers M, Meijer K, Hillege HL. Minor bleeds alert for subsequent major bleeding in patients using vitamin $\mathrm{K}$ antagonists. Br J Haematol 2011;153(04):508-514

69 van Rein N, le Cessie S, van Vliet IP, et al. Increased risk of major bleeding after a minor bleed during treatment with vitamin $\mathrm{K}$ antagonists is determined by fixed common risk factors. J Thromb Haemost 2016;14(05):948-952

70 van der Meijden PE, Bouman AC, Feijge MA, et al. Platelet dysfunction in thrombosis patients treated with vitamin $\mathrm{K}$ antagonists and recurrent bleeding. PLoS One 2013;8(05):e64112

71 Wagenvoord RJ, Deinum J, Elg M, Hemker HC. The paradoxical stimulation by a reversible thrombin inhibitor of thrombin generation in plasma measured with thrombinography is caused by alphamacroglobulin-thrombin. J Thromb Haemost 2010;8(06):1281-1289

72 Gribkova IV, Lipets EN, Rekhtina IG, et al. The modification of the thrombin generation test for the clinical assessment of dabigatran etexilate efficiency. Sci Rep 2016;6:29242

73 Dale B, Eikelboom JW, Weitz JI, et al. Dabigatran attenuates thrombin generation to a lesser extent than warfarin: could this explain their differential effects on intracranial hemorrhage and myocardial infarction? J Thromb Thrombolysis 2013;35(02): 295-301 